\title{
Simplified model to describe the dissociative recombination of linear polyatomic ions of astrophysical interest
}

\author{
N. Douguet ${ }^{1}$, S. Fonseca dos Santos ${ }^{1}$, V. Kokoouline ${ }^{2}$ and A.E. Orel ${ }^{1}$ \\ ${ }^{1}$ Department of Chemical Engineering and Materials Science, University of California, Davis, \\ CA 95616, USA \\ ${ }^{2}$ Department of Physics, University of Central Florida, FL 32816, USA
}

\begin{abstract}
We present results of a theoretical study on dissociative recombination of the $\mathrm{HCNH}^{+}, \mathrm{HCO}^{+}$and $\mathrm{N}_{2} \mathrm{H}^{+}$linear polyatomic ions at low energies using a simple theoretical model. In the present study, the indirect mechanism for recombination proceeds through the capture of the incoming electron in excited vibrational Rydberg states attached to the degenerate transverse modes of the linear ions. The strength of the non-adiabatic coupling responsible for dissociative recombination is determined directly from the near-threshold scattering matrix obtained numerically using the complex Kohn variational method. The final cross sections for the process are compared with available experimental data. It is demonstrated that at low collision energies, the major contribution to the dissociative recombination cross section is due to the indirect mechanism.
\end{abstract}

\section{Introduction}

Dissociative recombination (DR) of molecular ions is a collision process in which an incident electron is initially captured in a temporary state. Then, the excess of electron kinetic energy can be transferred to nuclear motion until the unstable neutral molecule fragments into atomic and/or molecular products. The exchange of energy between electronic and vibrational degrees of freedom can be mediated either through capture in doubly-excited dissociative resonant states of the neutral molecule, or through a series of rovibrationally excited Rydberg resonances, which manifests itself as a dense resonant structure in the cross section. The efficiency of the DR process at low energy is largely enhanced when the resonant state crosses the ionic ground state near its equilibrium geometry, leading to the so-called direct mechanism. However, when the dissociative resonance state does not cross the ground electronic state of the ion in the Frank-Condon region, the direct mechanism no longer dominates the DR process. In this case, there is still an open route for the dissociation of the molecular ion via the indirect mechanism. The electron can be temporarily captured into a rovibrationally excited Rydberg state of the neutral molecule which, if the auto-ionization lifetime is sufficiently long, would find a pathway to dissociate through a cascade of lower Rydberg states coupled to each other through non-adiabatic effects. The indirect mechanism has been shown to be efficient in numerous systems, such as $\mathrm{H}_{3}^{+}[1], \mathrm{HeH}^{+}[2,3]$, and $\mathrm{HCO}^{+}[4,5]$. On the other hand, recent theoretical work has demonstrated that the direct DR mechanism is not an

This is an Open Access article distributed under the terms of the Creative Commons Attribution License 4.0, which permits unrestricted use, distribution, and reproduction in any medium, provided the original work is properly cited. 
efficient mechanism at low energy in $\mathrm{HCNH}^{+}$[6] and $\mathrm{N}_{2} \mathrm{H}^{+}$[7]. In this study we investigate in more detail the indirect DR mechanism of these two polyatomic ions and revisit the process in $\mathrm{HCO}^{+}$using a better theoretical approach.

The DR reaction is an important process in cold plasmas and is also considered to be the dominant recombination mechanism in planetary ionospheres and interstellar clouds. Due to the possible existence of many resonant states and dissociative routes, DR represents a very complex molecular reaction. Indeed, in spite of the numerous theoretical and experimental advances that have been achieved over the years, DR still remains a process that is not yet fully understood, especially in the case of polyatomic molecules (a comprehensive and general review of the achievement and remaining challenges in the study of DR can be found in [8]). For instance, only recently an agreement between theoretical results and experimental data for the DR of the simplest polyatomic ion $\mathrm{H}_{3}^{+}$was finally achieved [1,9-11]. The theoretical approach was based on the multichannel quantum defect theory (MQDT) and it involved a multi-step and quite sophisticated theoretical treatment. The model considered explicitly all degrees of freedom and included the non-adiabatic couplings between Rydberg states. However, the key ingredient to reach agreement with the experimental DR rate coefficient was the inclusion of the Jahn-Teller effect [1], which drives strong vibronic couplings. Shortly after, a simplified model to treat DR of polyatomic ions was developed by Jungen and Pratt and first applied to the DR of $\mathrm{HCO}^{+}[12]$ and $\mathrm{H}_{3}^{+}$[13]. This model, also based on MQDT, is a simple method to study DR when non-adiabatic effects are dominant in the capture process and for which one can consider only the main process of capture of the electron in the first doubly degenerate vibrational state of the ion (via Jahn-Teller or RennerTeller non-adiabatic couplings) and one neglects autoionization. Keeping this simplified view of the process, we have added few improvements to this model. Our approach goes beyond the Jahn-Teller and Renner-Teller model Hamiltonians for the electron-ion interaction, which were employed in the studies mentioned above. In the present study, we do not use such model Hamiltonians and take into account all possible vibronic couplings. This approach applied to non-linear molecular systems has provided good agreement with experimental data for several molecular ions [14-16]. However, application of this model to linear molecular ions is more complicated, and we present in this work a description of the necessary improvements.

We have chosen to study the three molecular ions, $\mathrm{HCNH}^{+}, \mathrm{HCO}^{+}$and $\mathrm{N}_{2} \mathrm{H}^{+}$, mainly due to their astrophysical interest. The DR of $\mathrm{HCNH}^{+}$and its isomers is an important reaction since it can lead to the formation of the neutral molecules $\mathrm{HCN}$ and $\mathrm{HNC}$, which are precursors in the formation of the amino-acids, which, in their turn, are involved in the origin of life. A theoretical study of the DR in $\mathrm{HCNH}^{+}$could also shed some light at the discrepancy of reported values for the abundance ratio $[\mathrm{HNC}] /[\mathrm{HCN}]$. In fact, the literature reports values that range from 0.015 to $5[17,18]$ for observations in cold dark clouds. However, a recent study indicates that this ratio might have been overestimated in cold dark clouds and should be closer to one [19]. Controversies also exist in the DR mechanism of $\mathrm{HCNH}^{+}$, since a recent theoretical study by Hickman et al. [20] claimed that direct dissociation could occur at low energy. On the other hand, a more recent theoretical study by Ngassan and Orel [6] showed that the theoretical direct DR cross section is lower than the experimental value, which leaves room for speculation about the indirect mechanism contribution.

The molecular ion $\mathrm{N}_{2} \mathrm{H}^{+}$is among the most abundant polyatomic ions in the interstellar media (ISM) and serves as a tracer of dense interstellar region. However, the available literature on the DR of this molecular ion is scarce and, from the theoretical point of view, somehow incomplete. Several experiments on DR in $\mathrm{N}_{2} \mathrm{H}^{+}$have been made [21-23] producing somewhat different rate coefficients as shown on the right panel of Fig. 4. Theoretical studies conducted by Talbi [24] and later by Hickman et al. [7] suggested that the direct DR mechanism could occur through the second dissociative state. However, Ref. [7] found it to be a very inefficient process at low energy. In this work, we have calculated the DR cross section at a lower energy range $(0.001$ to $0.1 \mathrm{eV})$ of astrophysical relevance in the indirect DR picture. At a higher energy range, we have also computed the direct DR cross section 
using multiconfiguration time-dependent Hartree-Fock (MCTDH) treatment [25] in a two-dimensional potential energy surface (PES) taking into account the motion of $\mathrm{H}$ around the $\mathrm{N}_{2}$ dimer with the N-N bond kept frozen at its equilibrium value. The study of the direct mechanism of $\mathrm{N}_{2} \mathrm{H}^{+}$will be the subject of a future study.

After $\mathrm{H}_{3}^{+}$, the most abundant polyatomic ion present in the ISM is $\mathrm{HCO}^{+}$. It is also present in the Martian atmosphere [26]. The indirect DR mechanism of this ion has actually been studied extensively $[4,5,12,27,28]$, however the calculations still remain a factor 2-3 lower than the experimental values. On the view of the successful results obtained in the highly symmetric molecular ions $\mathrm{H}_{3} \mathrm{O}^{+}$[14], $\mathrm{CH}_{3}^{+}$[15] and $\mathrm{NH}_{4}^{+}$[16], we have included the $\mathrm{HCO}^{+}$in the present study in an attempt to produce a better theoretical DR cross-section for this molecular ion at low energies. Finally, other improvements compared to our previous reported works [27, 28] are (1) a larger scale $a b$ initio calculation, (2) the inclusion of more vibronic couplings, and (3) a more accurate description of the vibronic couplings between involved electronic states, obtained directly from the ab initio scattering matrix instead of fitting performed on the ab initio data.

\section{Theoretical approach}

The approach employed in this work is similar to the treatment used to calculate the DR cross section of highly symmetric ions, which has been described in $[13,16]$. Hence, we only present a brief overview of its elements in the following development.

The starting point of the model is the computation of the ab initio PES of the molecular target and its series of Rydberg energies. The molecular energies are thus obtained as a function of the position of the nuclei and the calculations are carried out using the code MESA [29]. Following Refs. [5, 30], the description of the vibrational dynamics in the simplified model developed by Jungen and Pratt [12, 13] considers the electronic capture as the decisive step in the DR mechanism neglecting auto-ionization; i.e., after the neutral molecule is formed all the flux is transferred into dissociation. Therefore, within this approximation, the DR cross section is an upper bound since it does not reflect the competition between dissociation and auto-ionization. Rotation of the molecular target is also neglected in the model, which accounts only for the electronic and vibrational degrees of freedom. However, note that Jungen and Pratt used parameters obtained from spectroscopic data, such that the effect of rotational motion is partially included in their approach. Another simplification is that the cross section is averaged over the energy interval between consecutive Rydberg resonances, leading to a constant probability of electronic capture. The consequence of the last two points is the structureless nature of the cross section, which does not exhibit the usual rotational resonance features. The last simplification is to approximate the nuclear vibrational wave function as harmonic, thus leading to an analytical expression for the final cross section [12]. The data needed to construct the smooth scattering matrix are quantum defects obtained from the $a b$ initio energies of excited Rydberg states. Once the asymptotic and short-distance (of the incident electron coordinate) regions are connected through the vibrational frame transformation, it results in a simple analytical formula for the indirect DR cross section for which an extended derivation can be found in [31]

$$
\langle\sigma\rangle=\frac{\pi^{3} \hbar^{2}}{m_{e} E_{e l}} \sum_{i} \sum_{e e^{\prime}}\left(\frac{\partial \mu_{e e^{\prime}}}{\partial q_{i}}\right)^{2} \Theta\left(\hbar w_{i}-E_{e l}\right) .
$$

In the above expression, the brackets indicate averaging of the cross section over Rydberg resonances, $E_{e l}$ is the asymptotic electronic energy, $m_{e}$ is the electron mass and $q_{i}$ represents the dimensionless normal coordinate related to the mass-scaled normal coordinate $Q_{i}$ by the relation $q_{i}=Q_{i} \sqrt{\mu_{i} \omega_{i} / \hbar}$, where $\omega_{i}$ and $\mu_{i}$ are the frequency and reduced mass of the $i$ th mode, respectively. The symbols $\mu_{e e^{\prime}}$ denote the elements of the quantum defect matrix, where the subscripts $e$ and $e^{\prime}$ label the initial and final electronic states that diagonalize the electronic Hamiltonian at the linear equilibrium geometry of the 
molecular ion. Each electronic state belongs to an irreducible representation $\Gamma$ in the $C_{\infty v}$ symmetry group of linear ions and has a projection $\Lambda$ of the angular momentum on the molecular axis. The Heaviside step function guarantees that the contribution to the cross section from the mode $q_{i}$ becomes zero when the electronic energy is greater than the corresponding vibrational threshold. Finally, note that in the present study, we only consider the contribution from degenerate modes in Eq. (1).

A model quantum defects matrix is given below to lowest order in the doubly degenerate mode. The matrix is expressed in the electronic basis at the equilibrium geometry of the molecule with corresponding quantum defects $\left\{\mu_{e}^{o}\right\}$ and irreducible representation $\Gamma=\left(\Sigma, \Pi^{\prime}, \Pi^{\prime \prime}, \Delta^{\prime}, \Delta^{\prime \prime}\right)$ (the primed and double-primed superscripts denote respectively symmetric and antisymmetric wave functions with respect to the molecular plane). The Renner-Teller coupling coefficients $\gamma_{k}$ between degenerate $\pi^{\prime}$ and $\pi^{\prime \prime}$ states appear on the diagonal. The quantity $\rho$ is simply given by $\rho^{2}=q_{x}{ }^{2}+q_{y}{ }^{2}$, where $q_{x}$ and $q_{y}$ are the $x$ - and $y$-components of the doubly degenerate transverse mode. Apart from the Renner-Teller coupling, only electronic states with $\Delta \Lambda= \pm 1$ can couple to first order in $\rho$ by symmetry. The strength of these couplings is represented by the parameters $\lambda_{k}$.

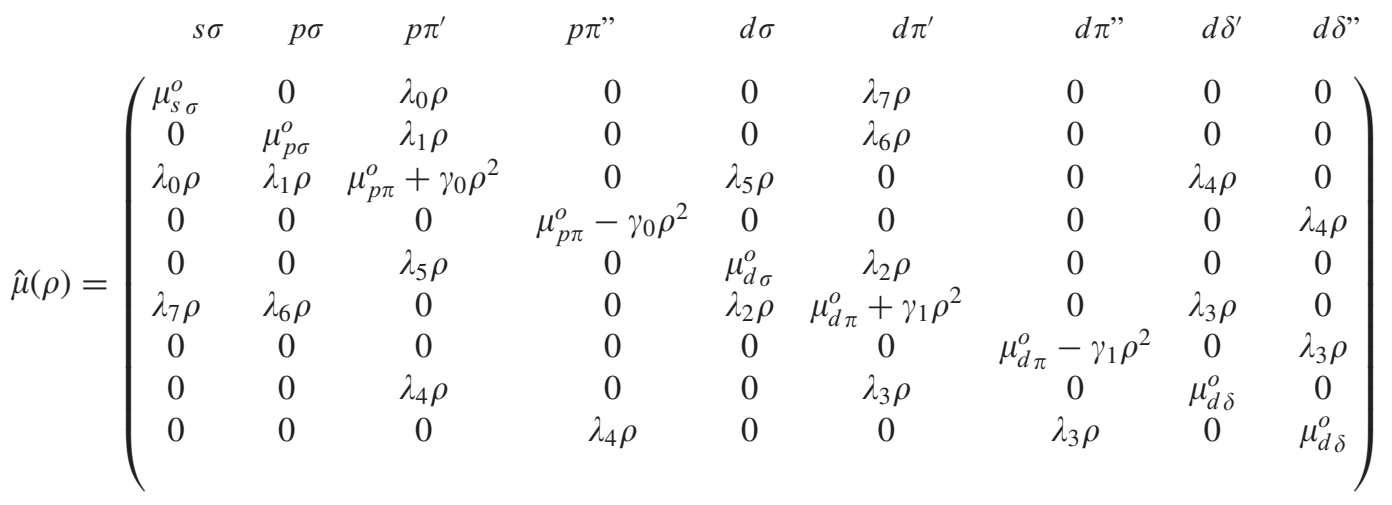

The electronic coupling coefficients between different electronic states is linear in $\rho$, while the Renner-Teller coupling has a quadratic dependence on the coordinate of distortion away from the ion's equilibrium geometry. Once diagonalized, the diagonal adiabatic energies shift quadratically with respect to $\rho$. This can be demonstrated, for instance, using perturbation theory [16]. Figure 1 shows this behavior for different electronic states in the case of the lowest degenerate mode of $\mathrm{HCNH}^{+}$. In the top panel, one can see the effective quantum numbers $(n=6)$ as functions of displacement along the normal mode component $Q_{1 x}$. Without zooming, the curves look flat. However, zooming in, as it is shown on two bottom panels, one sees the Renner-Teller splitting, as well as the electronic coupling between the non-degenerate states. On the lower left panel, the two $\Pi$ states demonstrate the Renner-Teller splitting. On the other hand (lower right panel), the Renner-Teller effect is perturbed by the interaction between the $s \sigma$ and $p \pi^{\prime}$ states, which clearly repel each other as $Q_{1 x}$ increases.

From the $a b$ initio energies, one can deduce the different parameters in the quantum defect matrix, for instance using a least-square procedure. However, this is a cumbersome approach in this case since one has to fit ten parameters and only eight quadratic coefficients are available. Furthermore, when two states are actually well spaced in quantum defect, interactions between Rydberg series with different principal quantum numbers can occur and this possibility is not reflected by this method. Finally, this model assumes that the quadratic diagonal coefficients vanish, a usual approximation, which is shown here to be poor for these linear polyatomic ions.

Alternatively, the cross section can be obtained directly via the low-energy scattering matrix calculated through the variational complex Kohn method (see, for instance, Refs. [32, 33]). In this approach, the scattering matrix is expressed in the spherical harmonic channel basis as $S_{l \Lambda, l^{\prime} \Lambda^{\prime}}(q)=$ $\left\langle l \Lambda|\hat{S}(q)| l^{\prime} \Lambda^{\prime}\right\rangle$, where $Y_{l \Lambda}(\theta, \phi)=\langle\theta \phi \mid l \Lambda\rangle$ are centered at a fixed origin in the molecular frame; 

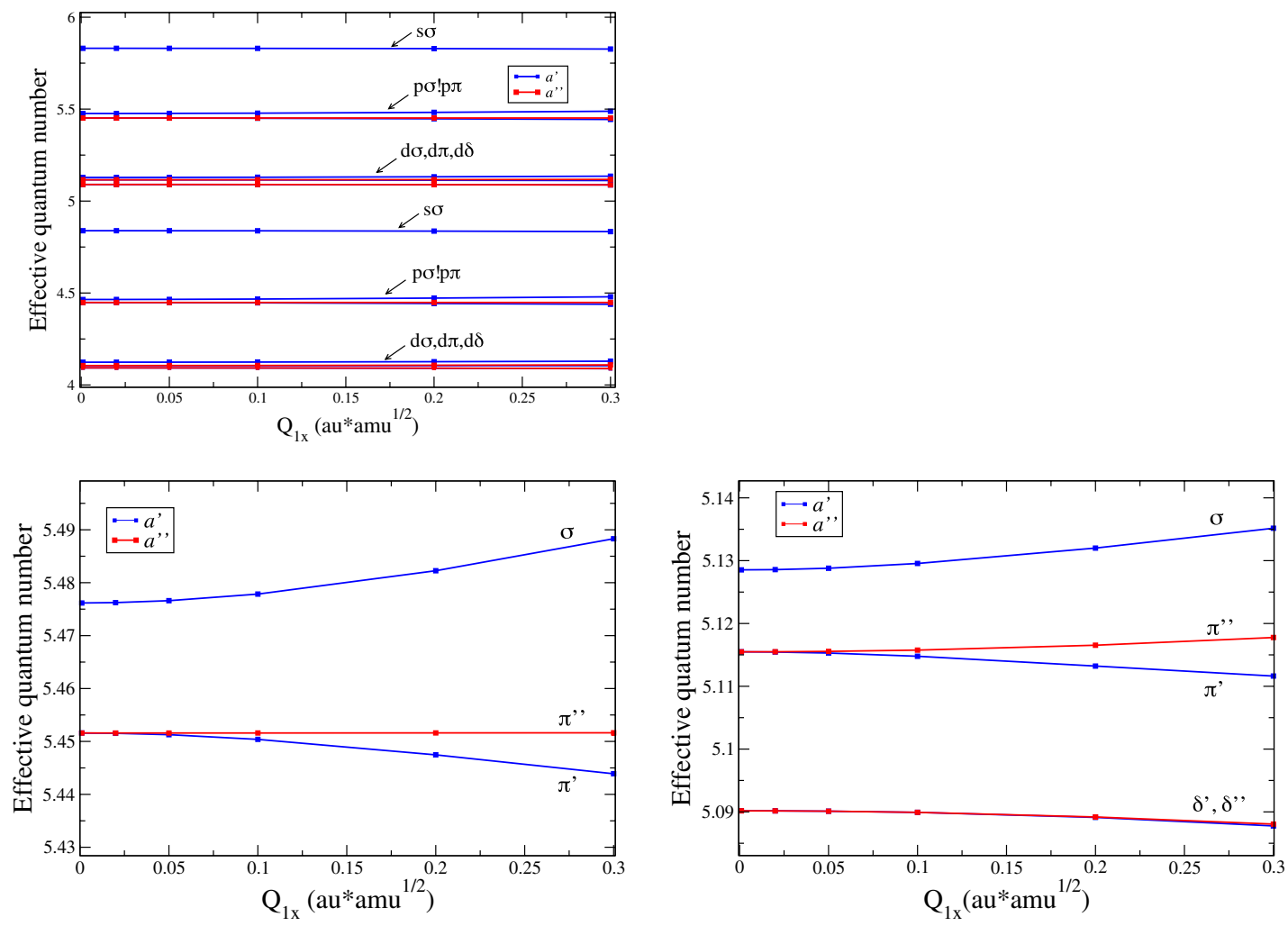

Figure 1. Coupling between Rydberg states of $\mathrm{HCNH}$. The upper panel contains the series of converged Rydberg states, while the two lower panels are enlarged areas of the upper panel. Lower left panel shows splittings between two different electronic states, $\mathrm{p} \sigma$ and $\mathrm{p} \pi$. Lower right panel shows the Renner-Teller splitting between $\mathrm{d} \pi$ states as well as interaction between $\mathrm{d} \pi^{\prime}$ and the $\mathrm{d} \sigma$ state above.

$(\theta, \phi)$ are polar angles. One can then transform the scattering matrix to the initial electronic basis $e$ and obtain an equivalent form of Eq. (1) as

$$
\langle\sigma\rangle=\frac{\hbar^{2} \pi}{4 m_{e} E_{e l}} \sum_{i} \sum_{e e^{\prime}}\left|\frac{\partial S_{e e^{\prime}}}{\partial q_{i}}\right|^{2} \Theta\left(\hbar w_{i}-E_{e l}\right) .
$$

As a test to verify the agreement between the results of our $a b$ initio energy and scattering calculations, we have extracted the quantum defects from the eigenphases of the scattering matrix given by the complex Kohn method using Seaton's theorem $\left(S=e^{2 i \pi \mu}\right)$ and compared these values against the $a b$ initio quantum defects calculated by MESA. The Rydberg states energies are calculated using the diffuse universal gaussian basis set $[8 s, 7 p, 6 d]$ optimized by Kaufmann et al. [34] to represent accurately Rydberg wave functions. Figure 2 shows the comparison between effective quantum number of $s \sigma$ and $p \pi$ electronic states of $\mathrm{N}_{2} \mathrm{H}^{*}$. Note that in Eqs. (1) and (2), the derivatives of quantum defects and elements of the scattering matrix determine the total cross section. The agreement between the derivatives of quantum defects and elements of the scattering matrix is very good, indicating the high precision of the scattering matrix and quantum defect calculations.

The strength of the electronic coupling between different electronic states can be directly expressed in terms of the geometry dependence of the scattering matrix elements. Table 1 shows the values of the largest coupling elements for $\mathrm{N}_{2} \mathrm{H}$ and $\mathrm{HCO}$. One can see that for both molecules, the $s \sigma-p \pi^{\prime}$ couplings 

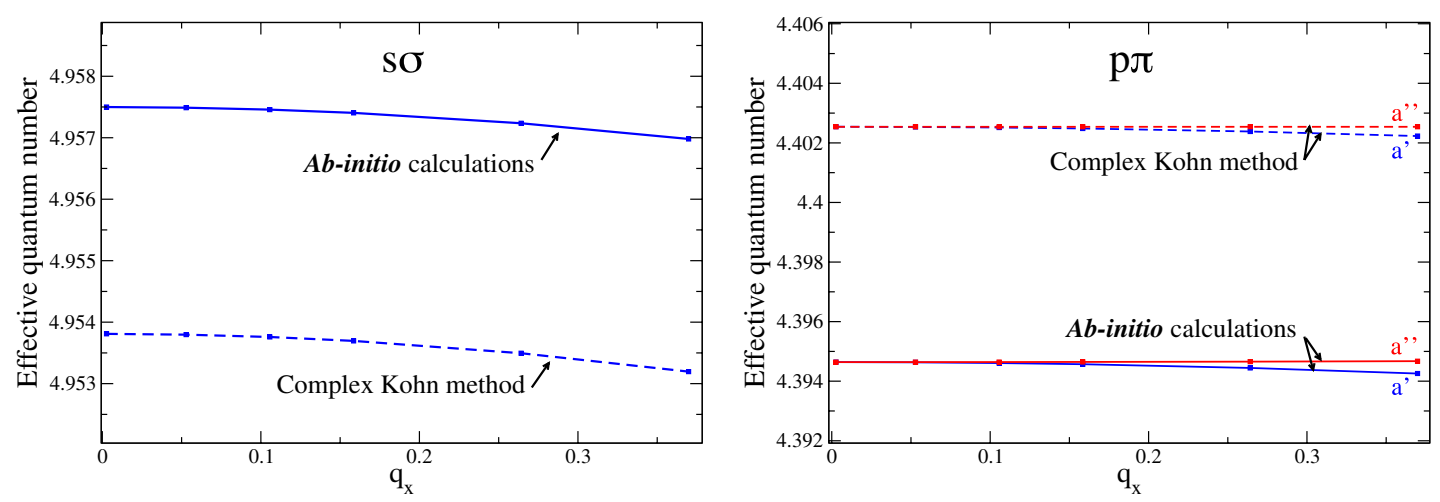

Figure 2. Comparison between geometry-dependent effective quantum numbers obtained from our ab initio calculations and from the phase shifts obtained in the scattering calculations as a function of the $q_{x}$ component of the transverse mode. Left and right panels show, respectively, the quadratic dependence of the $s \sigma$ state of $\mathrm{HCO}^{*}$ and the splitting of the $p \pi$ states of $\mathrm{N}_{2} \mathrm{H}^{*}$.

Table 1. The left table contains the most important electronic couplings between electronic states $e-e^{\prime}$ obtained from variation of the scattering matrix element with respect to the transverse normal mode component $q_{x}$ for $\mathrm{HCO}$ while the right table shows the same information for $\mathrm{N}_{2} \mathrm{H}$.

\begin{tabular}{|c|c|c|c|}
\hline$e-e^{\prime}$ & $\frac{\partial S_{e e^{\prime}}}{\partial q_{x}}$ & $e-e^{\prime}$ & $\frac{\partial S_{e e^{\prime}}}{\partial q_{x}}$ \\
\hline$s \sigma-p \pi$ & 0.12 & $s \sigma-p \pi$ & 0.18 \\
$p \pi-d \delta$ & 0.07 & $p \sigma-p \pi$ & 0.15 \\
$d \pi-d \delta$ & 0.06 & $p \pi-d \delta$ & 0.11 \\
$s \sigma-d \pi$ & 0.06 & $s \sigma-d \pi$ & 0.07 \\
$p \sigma-d \pi$ & 0.04 & $d \pi-d \delta$ & 0.06 \\
\hline
\end{tabular}

are the most important. This coupling was neglected in previous studies [5,27], which explains partially the difference between cross sections obtained in Refs. [5, 27] and in this study, which is demonstrated in Fig. 5.

\section{Results}

The DR cross section of $\mathrm{HCNH}^{+}$was evaluated solely from the ab initio calculation using Eq. (1) by fitting the parameters of the quantum defect matrix to the bound state energy calculations (the complete scattering calculation will be performed in a future study). Figure 3 shows the theoretical cross section in comparison with the experimental results by Semaniak et al. [35]. One interesting feature of $\mathrm{HCNH}^{+}$is the presence of two transverse normal modes with very different asymmetrical elongations of the hydrogen atoms on both sides of the molecular ion. This characteristic of the ion is reflected by the double drop in the theoretical cross section, the first one corresponding to the mode with frequency $\omega_{1}=642 \mathrm{~cm}^{-1}$ and the second drop corresponding to $\omega_{2}=801 \mathrm{~cm}^{-1}$. There is a good agreement between theoretical and experimental results. Nevertheless, the accurate $\mathrm{HCO}^{+}$and $\mathrm{N}_{2} \mathrm{H}^{+}$scattering calculations indicate that the cross section obtained from fitting the $a b$ initio quantum defects has a tendency to overestimate the value of the theoretical cross section in linear polyatomic ions.

Figure 4 shows the DR cross section (left panel) and rate coefficient (right panel) for $\mathrm{N}_{2} \mathrm{H}^{+}$ calculated using the complex Kohn method. The disagreement between theory and experiment is noticeable at energy well below the first vibrational threshold. On the other hand, as one can see on 


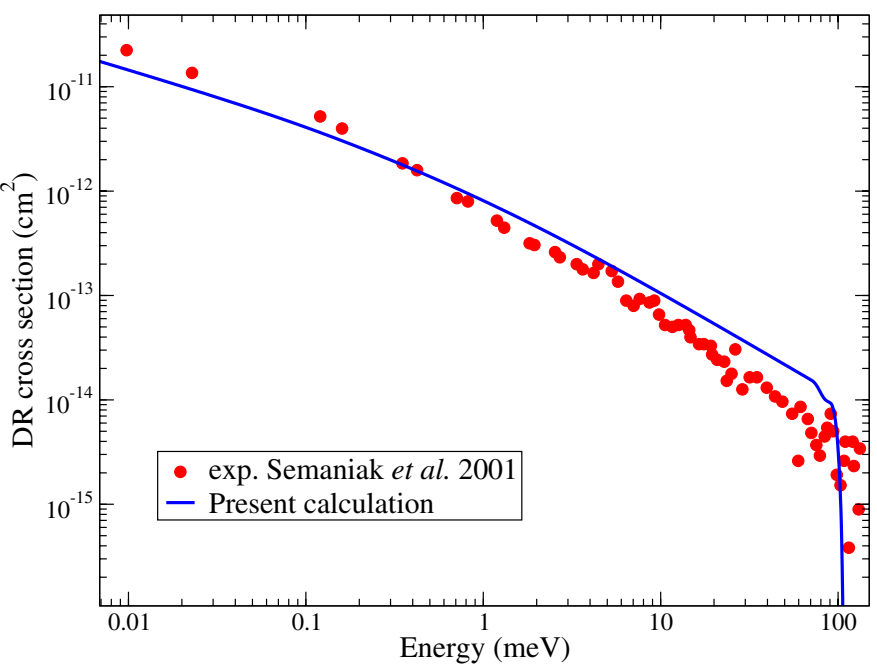

Figure 3. Theoretical DR cross section of $\mathrm{HCNH}^{+}$(solid blue line) obtained in this study compared to the experimental result (red dots) from Ref. [35].
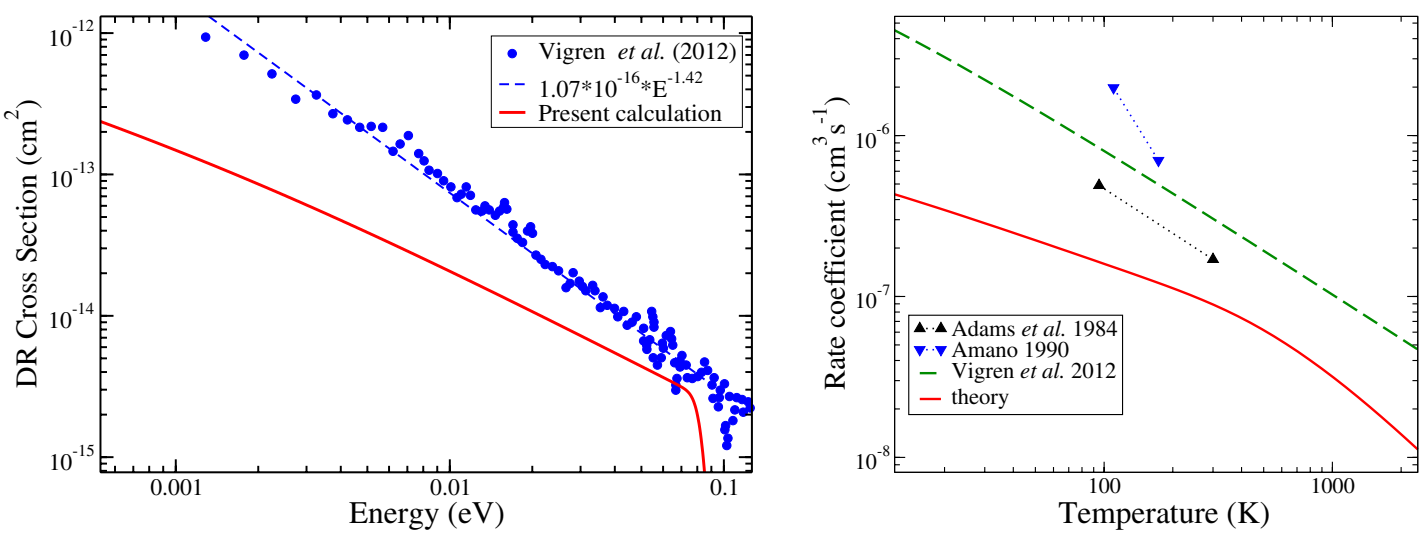

Figure 4. On the left side, theoretical (solid red line) and experimental (dots) DR cross section from the CRYRING storage ring experiment [23] of $\mathrm{N}_{2} \mathrm{H}^{+}$. The dashed line is an $E^{\alpha}$ fit to the experimental curve given in [23]. On the right side, theoretical rate coefficients compared with different experimental measurements [21-23].

the right panel of Fig. 4, the rate coefficients obtained in different experiments [21-23] do not agree well with each other. Although we do not have an explanation for the disagreement, it is worth to mention that the curve fitting the CRYRING storage ring experimental data [23] (dashed curve in left panel) does not behave as $E_{e l}^{-1}$ as expected from the Wigner threshold law (The theoretical cross section does obey the Wigner law). Furthermore, preliminary calculations are being performed including not only the transverse doubly degenerate mode, but also the two longitudinal stretching modes of $\mathrm{N}_{2} \mathrm{H}^{+}$. These results are so far promising and we believe a much better agreement will be achieved once these modes are included in the model. However, it will not still not be possible to explain the $E_{e l}^{-1.46}$ behavior of the experimental data using our model and could probably be explained in part by the high rotational temperature in the experiment. 


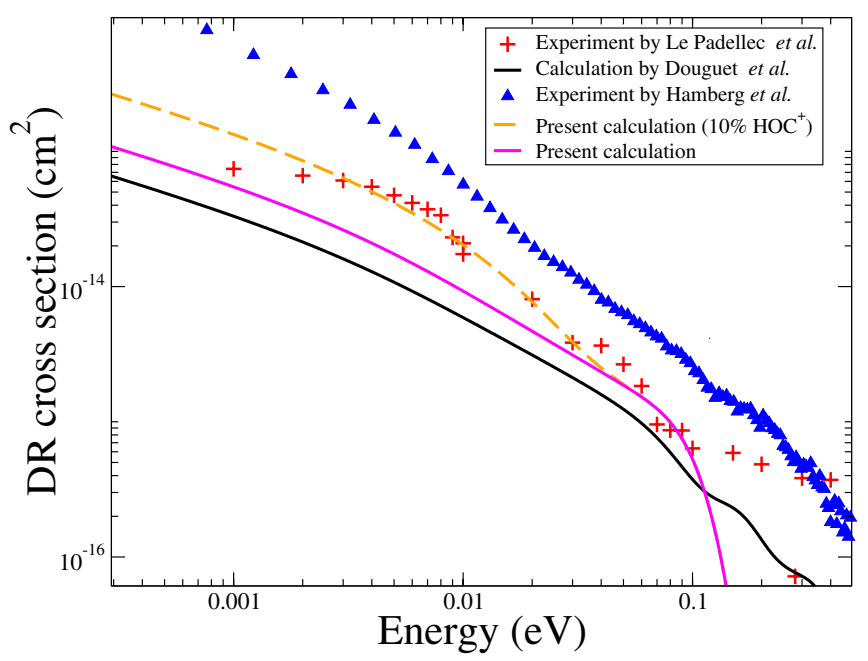

Figure 5. $\mathrm{HCO}^{+} \mathrm{DR}$ cross section. The cross sections of the present study are shown by the magenta solid line, obtained for pure $\mathrm{HCO}^{+}$and by the orange dashed line obtained for a $90 \%-10 \%$ mixture of $\mathrm{HCO}^{+}$and $\mathrm{HOC}^{+}$ isomers. Also shown in black solid line, the data of a previous theoretical study by Douguet et al. [27]. The experimental data are from a merged beam experiment of Le Padelec et al. [36] and the CRYRING storage ring experiment of Hamberg et al. [37]. Previous theoretical cross sections are also shown for comparison.

Finally, Fig. 5 shows the different theoretical and experimental results for the DR cross section of $\mathrm{HCO}^{+}$. In this present study, we have calculated the cross section for $\mathrm{HCO}^{+}$(solid magenta line) as well as a cross section assuming $10 \%$ of contribution from the $\mathrm{HOC}^{+}$isomer (orange dashed line). For both cases, there is an overall improvement in agreement with the experiment comparing to our previous models [5, 27] as well as to the results of Jungen and Pratt [12], especially if we compare our results with the flowing afterglow experiment by Le Padellec et al. [36]. Both experimental cross sections present a drop at about $840 \mathrm{~cm}^{-1}$, which corresponds to the vibrational threshold of the doubly degenerate transverse mode of $\mathrm{HOC}^{+}$. A better agreement with experiment is achieved if we assumes that there is $10 \%$ and $90 \%$ of $\mathrm{HOC}^{+}$and $\mathrm{HCO}^{+}$isomers in the ion source, suggesting that there might be some percentage of the $\mathrm{HOC}^{+}$isomer in experiments, as was suggested to us by R. Johnsen. The HOC ${ }^{+}$ isomer has a very low degenerate-mode frequency $\left(150 \mathrm{~cm}^{-1}\right)$, which might explain the shoulder-like feature in the experimental cross section at energy near $10 \mathrm{meV}$. However, our $\mathrm{HCO}^{+} \mathrm{DR}$ cross section stills shows a noticeable disagreement with the data of the CRYRING storage ring experiment (blue triangle) which disagrees with Le Padellec et al. experimental data: the cross section is about factor 2-3 higher. We are currently carrying out calculations including the two longitudinal modes of $\mathrm{HCO}^{+}$ and we anticipate a general increase of the cross section due to the inclusion of these modes, which should bring the theoretical results in closer agreement with the CRYRING data.

\section{Conclusions}

We have studied the low-energy DR of the linear molecular ions $\mathrm{HCNH}^{+}, \mathrm{N}_{2} \mathrm{H}^{+}$and $\mathrm{HCO}^{+}$using a simple theoretical model and assuming initial capture though degenerate transverse modes. In the case of $\mathrm{HCO}^{+}$and $\mathrm{N}_{2} \mathrm{H}^{+}$, the non-adiabatic couplings are evaluated using the scattering matrix calculated via the complex Kohn method. The accuracy of the scattering matrix is verified by comparing the eigenphases in the scattering calculations to ab initio quantum defects. A good agreement is found. 
The cross sections agree relatively well with experimental data for the three considered molecules, but the theoretical results are somewhat below the experimental data. The longitudinal modes of the ions have not been taken into account in this study, however, we believe that they could play an important role in the DR process and, once included, might bring the theoretical results even closer to the experimental measurements.

This work is supported by the DOE Office of Basic Energy Science and the National Science Foundation, Grant No's PHY-11-60611 and PHY-10-68785.

\section{References}

[1] Kokoouline V, Greene C H and Esry B D 2001 Nature 412, 891

[2] Guberman S L 1994 Phys. Rev. A 49, R4277

[3] Haxton D J and Greene C H 2009 Phys. Rev. A 79, 022701

[4] Larson A, Tonzani S, Santra R and Greene C H 2005 J. Phys.: Conf. Ser. 4, 148

[5] Mikhailov I A, Kokoouline V, Larson A, Tonzani S and Greene C H 2006 Phys. Rev. A 74, 032707

[6] Ngassam V and Orel A E 2007 Phys. Rev. A 75, 062702

[7] Hickman A P, Kashinski D O, Malenda R F, Gatti F and Talbi D 2011 Journal of Physics: Conference Series 300, 012016

[8] Larsson M and Orel A 2008 Dissociative Recombination of Molecular Ions (Cambridge University Press)

[9] Kokoouline V and Greene C H 2003 Phys. Rev. Lett. 90, 133201

[10] Kokoouline V and Greene C H 2003 Phys. Rev. A 68, 012703

[11] Fonseca dos Santos S, Kokoouline V and Greene C H 2007 J. Chem. Phys. 127, 124309

[12] Jungen C and Pratt S T 2008 J. Chem. Phys. 129, 164311

[13] Jungen C and Pratt S T 2009 Phys. Rev. Lett. 102, 023201

[14] Douguet N, Orel A, Mikhailov I, Schneider I F, Greene C H and Kokoouline V 2011 J. Phys.: Conf. Series 300, 012015

[15] Douguet N, Orel A E, Greene C H and Kokoouline V 2012 Phys. Phys. Lett. 108, 023202

[16] Douguet N, Kokoouline V and Orel A E 2012 J. Phys. B: At. Mol. Opt. Phys. 45, 051001

[17] Goldsmith P F, Langer W D, Elder J, Irvine W and Kollberg E 1981 Astrophys. J. 249, 524

[18] Wootten A, Evans II N J, Snell R and Bout P V 1978 Astrophys. J. 225, L143

[19] Sarrasin E, Abdallah D B, Wernli M, Faure A, Cernicharo J and Lique F 2010 MNRAS 404, 518

[20] Hickman A P, Miles R D, Hayden C and Talbi D 2005 Astron. Astrophys. 31, 438

[21] Adams N G and Babcosk L M 1994 J. Phys. Chem. 98, 4564

[22] Amano T 1990 J. Chem. Phys. 92, 6492

[23] Vigren E, Zhauerchyk V, Hamberg M, Kaminska M, Semaniak J, af Ugglas M, Thomas R D and Geppert W D 2012 Astrophys. J. 757, 34

[24] Talbi D 2006 Chem. Phys. 332, 298-303

[25] Worth G A, Beck M H, Jäckle A and Meyer H D 2012 The MCTDH Package, Version 8.3 http://www.pci.uni-heidelberg.de/tc/usr/mctdh

[26] Krasnopolsky V A 2002 J. Geophys. Res. 107, 5128

[27] Douguet N, Kokoouline V and Greene C H 2008 Phys. Rev. A 77, 064703

[28] Douguet N, Kokoouline V and Greene C H 2009 Phys. Rev. A 80, 062712

[29] Saxe P, Lengsfield B H, Martin R and Page M 1990 MESA (Molecular Electronic Structure Applications)

[30] Kokoouline V, Douguet N and Greene C H 2011 Chem. Phys. Lett. 507, 1

[31] Fonseca dos Santos S, Douguet N, Kokoouline V and Orel A E 2014 J. Chem. Phys. 140, 164308

[32] C W McCurdy and T N Rescigno 1989 Phys. Rev. A 39, 4487 
[33] Orel A E, Rescigno T and Lengsfield B 1991 Phys. Rev. A 44, 4328

[34] Kaufmann K, Baumeister W and Jungen M 1989 J. Phys. B: At. Mol. Opt. Phys. 22, 2223

[35] Semaniak J et al 2001 Astrophys. J. Suppl. Ser. 135, 275

[36] LePadellec A, Sheehan C, Talbi D and Mitchell J B A 1997 J. Phys. B: At. Mol. Opt. Phys. 30, 319

[37] Hamberg M et al 2014 J. Phys. Chem. A 118, 6034 